\title{
IMPACTOS MEDIOAMBIENTALES Y SOCIALES DEL TRANSPORTE
}

\author{
José GARRIDO \\ Departamento de Geografía y Ordenación del Territorio \\ Universidad de Zaragoza
}

\begin{abstract}
Resumen: Este trabajo estudia la interacción entre el transporte y el medio ambiente, considerando los impactos del primero sobre el entorno y la salud pública.
\end{abstract}

Palabras clave: Transporte, medio ambiente, movilidad, ecología, energía.

\begin{abstract}
This work studies the interaction between transport and environment, considering the impact of the former in the surroundings and the public health.
\end{abstract}

Key words: Transport, environment, mobility, ecology, energy.

\section{INTRODUCCIÓN}

La interacción mutua de transporte y medio ambiente constituye una preocupación creciente en el mundo actual. El efecto invernadero y la reducción de la capa de ozono son algunos de los ejemplos que expresan ese carácter universal. El reconocimiento de la preocupante situación se manifiesta en Documentos como Transport 2000 Plus (1991), el Libro Verde sobre el Impacto Medioambiental del Transporte (1992), el V Programa de Acción sobre el Medio Ambiente (1992) o el Informe para la Construcción de un Marco Comunitario que garantice la Movilidad Sostenible (1993), así como la Cumbre de la Tierra.

Por su parte, la incidencia de los impactos nocivos del transporte rebasa los límites del entorno para afectar a la propia salud humana y por supuesto al desarrollo de las regiones. En este trabajo no pretendemos abordar el conjunto de los problemas que comporta la actividad del transporte, ni señalar todas las soluciones que pueden ofrecerse en este tema, sino tan sólo queremos enfatizar aquellos puntos de mayor trascendencia de esa interacción y proponer algunas tendencias ad hoc. 
En una primera parte se acometen aspectos conceptuales del transporte desde la perspectiva del medio ambiente, cuestionando la demanda constante de infraestructuras o la propia movilidad y estableciendo el límite del crecimiento de la actividad. Más tarde se tratan los impactos ambientales y sociales del transporte.

\section{EL TRANSPORTE DESDE LA PERSPECTIVA DEL MEDIO AMBIENTE}

Si bien la preocupación del transporte en Europa se remonta a la década de los setenta, en España es más reciente, en torno a mediados de los ochenta, debido fundamentalmente a dos factores:

- La creciente preocupación ambiental de la sociedad

- El desarrollo del marco legislativo

En cualquier caso e independientemente del ámbito espacial y de la época de su interés, el transporte y el medio ambiente constituyen dos elementos que deben entenderse, limitando así la creciente motorización que altera el entorno y reflexionando sobre los principios de actuación de la economía ecológica del transporte que complementa los modelos de la economía tradicional.

\section{Transporte versus medio ambiente}

Se comenta que el transporte constituye un «problema» para el medio ambiente cuando en realidad su génesis está en la propia población que demanda ese servicio. Ahora bien, más que dos variables opuestas que se alejan y viajan separadas en el tiempo y en el espacio, hay que intentar aunarlas, que se respeten y que sus efectos nocivos sean los mínimos posibles.

Sin embargo, el desarrollo del transporte es considerado, en sentido amplio, como sinónimo de riqueza, progreso y como un bien en sí mismo que debe potenciarse. Buena parte de la población clama por más infraestructuras y de mayor calidad, medios de transporte potentes y rápidos, y mayor volumen de servicios de transporte que facilite el desplazamiento de personas y mercancías.

Junto a ello, las regiones y ciudades compiten en el Olimpo nacional por atraer hacia sí nuevas autopistas, autovías, el AVE o la captación de pasajeros o mercancías. Se trata, pues, de escalar posiciones en el ranking nacional e internacional donde se puja por la mayor consideración de región o ciudad desarrollada. 
Esta es la clara situación de Aragón, dado que además de la justificada autovía NorteSur en construcción, se reclama el paso del AVE para el año 2002, a ser posible con paradas en la Estación Intermodal y en el Aeropuerto, y se exige la implantación de una plataforma logística que dinamice el complejo aeronáutico e impulse el tráfico aéreo a cotas elevadas en el marco nacional.

Esta es la situación de la Comunidad aragonesa y esos son los deseos de buena parte de la población, de los diferentes partidos políticos y de los principales agentes sociales. Pero frente a ello cabe preguntarse si realmente esta demanda de infraestructuras y medios de transporte tiene límite $\mathrm{o}$, mejor aún, si el sacrificio ambiental puede soportar tales peticiones.

Es habitual, por otro lado, minorar los efectos perniciosos de aquellos elementos que tanto anhelan, y sobre todo cuando se acompañan de la ciencia y tecnología ya experimentadas en países más desarrollados. Es decir, el esplendor de las nuevas infraestructuras justifica sobremanera cualquier objeción o impacto negativo.

No obstante, desde el punto de vista ecológico el sistema de transporte ilimitado choca frontalmente con el fraccionamiento de los ecosistemas (BARKE, 1986). En efecto, de la energía irradiada por el Sol solamente una parte es absorbida por las plantas; mediante la fotosíntesis esa energía solar es transformada en energía química, la cual se distribuye en el propio desarrollo de los vegetales, en el alimento de los herbívoros y en la constitución de la biomasa vegetal cuando las hojas caen o se muere la planta (MARGALEF, 1992).

En cualquier caso, lo relevante es el hecho de que una pequeña parte de esa energía pasa a los niveles tróficos de los consumidores y que aquella debe dosificarse adecuadamente para su supervivencia. Estas son, pues, las dos ideas clave que queremos destacar: por un lado, la parte sustancial de la biomasa y de la energía se localiza en el reino vegetal; y, por otro, la energía disponible en los consumidores es muy limitada y precisa una gestión correcta.

En esta línea queremos situar el transporte, pues la expansión de la denominada «Triple A» del transporte - Automóvil, AVE y Avión- requiere cada día mayor cantidad de energía, la cual es escasa, y se contrapone al funcionamiento de los ecosistemas terrestres. Por su parte, la construcción de nuevas infraestructuras reduce el espacio ocupado por la Naturaleza, fracciona el hábitat de las diferentes especies y limita la supervivencia de muchos seres vivos.

Además, las altas tasas de motorización exigen la utilización de elevadas cantidades de materiales y energía (TOLLEY, 1995). Precisamente algunos de los materiales necesarios para la construcción de los medios de transporte se encuentran en situación «crítica» o son muy escasos sobre la Tierra. Asimismo, la generalización del transporte produce enormes masas de residuos sólidos, líquidos y gaseosos que la Naturaleza es incapaz de digerir. 
Ejemplos comunes de esta situación son las emisiones masivas de $\mathrm{CO}_{2}$ a la atmósfera, el vertido de petróleo en los mares y los vehículos desechados que se acumulan en el mejor de los casos en vertederos controlados. Cuando las cantidades son pequeñas el ecosistema global es capaz de absorber y autodepurar los efectos nocivos, pero cuando la degradación alcanza ciertos niveles el proceso es exponencial y se acerca rápidamente a situaciones de ruptura (MEADOWS, 1985)

\section{Los límites ambientales}

El concepto que mejor define el límite ambiental es el de capacidad de carga, entendida como la "cantidad total de transporte que un ecosistema natural puede soportar sin superar cierto umbral de deterioro» (ESTEVAN, 1996). En otras palabras, la contradicción que existe entre el crecimiento ilimitado del transporte y el medio ambiente tiene su máxima expresión en la ruptura del equilibrio ecológico.

Esta preocupación por el crecimiento ilimitado del transporte se constata en el Primer Proyecto Oficial de la Unión Europea, dado que una de las soluciones estratégicas que postula es la de «réduire le taux de croissance, spécialement en reflétant correctement dans les prix la facture environnementale du transport» (COMISIÓN DE LAS COMUNIDADES EUROPEAS, 1997).

Ahora bien, el límite ambiental no es estático, pues la tecnología y la organización del transporte pueden conseguir que la capacidad de carga sea más elevada. Por ejemplo, la introducción de catalizadores en los vehículos puede reducir la emisión de plomo a la atmósfera, lo cual facilita que mayor número de automóviles puedan circular por el mismo ecosistema sin rebasar los límites de plomo establecidos.

De igual manera se puede pensar en un ecosistema urbano, por ejemplo Zaragoza, donde el perfeccionamiento de la organización del tráfico con la construcción de los Cinturones de Ronda comportan mayor dispersión de la carga contaminante, en particular alivia esa carga en unos puntos concretos de la ciudad donde precisamente reside gran parte de la población. Asimismo, si la congestión de tráfico es menor el volumen total de emisiones también se reducirá.

Por tanto, está claro que el límite ambiental se eleva con relación a la tecnología y la organización, pero como todo fenómeno físico estả afectado por la ley de los rendimientos decrecientes. De hecho, si se aumenta la velocidad en los desplazamientos, a igualdad de las demás condiciones físicas implícitas en el movimiento, sólo puede lograrse a costa de mayores consumos de energía y también de los diversos materiales utilizados en la construcción de vehículos e infraestructuras, de forma que el incremento de la velocidad seguirá ofreciendo rendimientos decrecientes en relación con esos consumos. 
Así pues, la capacidad de carga de un ecosistema presenta unos límites absolutos que nunca han de superarse. Si se aumenta el transporte de forma ilimitada, si se incrementa la velocidad, o ambas a la vez, en un determinado ecosistema, éste se deteriorará progresivamente y las medidas tecnológicas y organizativas únicamente lograrán frenar o moderar el proceso pero no invertirlo.

Esta es la clave del problema del transporte y el medio ambiente, ya que sitúa el equilibrio ecológico en primer término de los intereses humanos a largo plazo, pero se enfrenta a los intereses inmediatos del transporte. En cualquier caso, esa concepción aporta un marco general de actuación pero no la comprensión global del dilema ambiental del transporte.

Quizá sea menester recordar que el deterioro ambiental se ha acentuado tras el inicio de la revolución industrial, habida cuenta que las nuevas tecnologías han aumentado la carga del transporte y la velocidad (WOLKOWITSCH, 1992). Por contra, ese desarrollo tecnológico no ha conseguido frenar la afección al medio ambiente, pese a que se ha potenciado la sensibilización ambiental en los últimos años.

La conciencia ambiental y la situación de la carga ambiental del transporte, la cual ha superado límites socialmente admisibles en determinadas regiones o ecosistemas urbanos del mundo desarrollado, ha supuesto que en determinados niveles de la Administración se hayan adoptado medidas para afrontar esta problemática.

Un ejemplo ilustrativo de esto se muestra en el Informe Transport 200 Plus: el transporte en Europa en rápida transformación, pues se expone que «desde hace algunos años Europa parece haber sobrepasado el punto más allá del cual cualquier incremento del tráfico es contraproducente. La suma de efectos negativos parece cancelar los incrementos de riqueza, eficiencia, confort y facilidad que deberían resultar del crecimiento del volumen de tráfico».

Al igual que en Europa, en el ámbito nacional y regional tratan de adaptarse a la normativa de la Unión Europea y asumir las mismas preocupaciones en materia medioambiental, traduciéndose para el transporte en la necesidad de reducir, moderar o estabilizar el volumen total de tráfico y la demanda de transporte como única forma de contener los efectos negativos de esta actividad.

\section{IMPACTOS MEDIOAMBIENTALES DEL TRANSPORTE}

La estrategia del Banco Mundial para todos los países es promocionar políticas de transporte y apoyar proyectos que ayuden a frenar el deterioro ambiental y reducir el nivel global de emisiones. En la misma línea, el V Programa de Acción sobre el Medio Ambiente de la Unión Europea concentra su actividad en cinco sectores económicos principales, siendo 
uno de ellos precisamente el transporte, y determina para el año 2000 el cumplimiento de una serie de objetivos en función de los agentes contaminantes. Así pues, veamos la situación del transporte y la interacción de éste con el consumo de energía.

\section{Situación del transporte}

El transporte ha experimentado un crecimiento muy intenso en las últimas décadas. Así, desde 1970 la tasa de crecimiento anual en la Unión Europea ha sido del 3,1 \% en el tráfico de pasajeros y del 2,3\% en mercancías (CÁDIZ, 1994), de modo que las previsiones entre el año 1990 y el 2010 señalan que el transporte por carretera de mercancías se duplicará y el de pasajeros se incrementará alrededor del $50 \%$.

Tabla 1. Tráfico Interior de viajeros en España (millones viajeros $/ \mathrm{km}$ )

\begin{tabular}{|lrrrrrr|}
\hline Modos & Total (1980) & \multicolumn{1}{c}{$\%$} & Total (1985) & \multicolumn{1}{c}{$\%$} & Total (1993) & \multicolumn{1}{c|}{$\%$} \\
\hline Carretera & 198.217 & 90,12 & 179.232 & 88,56 & 231.109 & 89,4 \\
Ferrocarril & 14.826 & 6,74 & 17.038 & 8,42 & 17.624 & 6,8 \\
Aéreo & 5.762 & 2,62 & 5.216 & 2,58 & 8.642 & 3,3 \\
Marítimo & 1.126 & 0,51 & 888 & 0,44 & 1.200 & 0,5 \\
\hline
\end{tabular}

Fuente: Piñeiro (1990) y MOPTMA (1994)

Tabla 2. Tráfico Interior de mercancías en España (millones de tm/ $\mathrm{km}$ )

\begin{tabular}{|lcrrrrr|}
\hline Modos & Total (1980) & \multicolumn{1}{c}{$\%$} & Total (1985) & \multicolumn{1}{c}{$\%$} & Total (1993) & \multicolumn{1}{c|}{$\%$} \\
\hline Carretera & 98.898 & 68,480 & 120.045 & 73,02 & 160.000 & 78,8 \\
Ferrocarril & 11.300 & 7,83 & 11.906 & 7,24 & 9.550 & 4,7 \\
Aéreo & 74 & 0,05 & 60 & 0,03 & 96 & 0,4 \\
Marítimo & 31.125 & 21,55 & 29.299 & 17,78 & 32.711 & 16,1 \\
\hline
\end{tabular}

Fuente: Piñeiro (1990) y MOPTMA (1994)

A su vez, la distribución modal del transporte pone de relieve la preeminencia del tráfico rodado, tanto de pasajeros como de mercancías, con porcentajes que alcanzan el 89,4 $\%$ y $78,8 \%$, respectivamente. Por su parte, los valores del resto de los modos de transporte distan mucho de los umbrales mencionados.

El reparto modal no ha variado sustancialmente en los últimos años, aunque se aprecia una tendencia expansiva del tráfico aéreo en detrimento del ferrocarril y marítimo. No obstante, el Tren de Alta Velocidad puede ser un competidor significativo para la carretera 
y el transporte aéreo para distancias superiores a los $300 \mathrm{~km}$., pues la facilidad del transporte interurbano y la rapidez del desplazamiento (velocidades medias de $250 \mathrm{~km}$ /hora) hacen muy eficaz este modo de transporte. Igualmente el ferrocarril se plantea como un modo sustitutivo a la carretera en los corredores donde hay problemas de congestión, como es el caso de las cercanías, si bien sólo existen en algunas ciudades.

\section{Transporte y energía}

Los diferentes modos de transporte se caracterizan por ser grandes consumidores de energía ${ }^{1}$, siendo uno de los subsectores que más contribuye al deterioro ambiental. Además, los productos energéticos que se utilizan son, en su mayor parte, de energías no renovables, de forma que el $65 \%$ de la energía consumida por el transporte procedía de los derivados del petróleo en 1990, y la tendencia es de incrementarse hasta el $75 \%$ a medio plazo.

En España, la participación de los transportes en el consumo de energía final fue del $33 \%$ en 1980 y del $42 \%$ en 1990, cuyos porcentajes son superiores a la Unión Europea, ya que alcanzaron unos valores del $25 \%$ y el $33 \%$ durante el mismo período.

Según un estudio realizado en la Unión Europea sobre el consumo de energía por modos de transporte (MOPT, 1992), el resultado revela que la carretera consume el $84,4 \%$ del total ${ }^{2}$, el transporte aéreo el $11,1 \%$, el ferrocarril el 2,5\% y la navegación fluvial el $2 \%$.

Sin embargo, esos valores son globales para el conjunto de vehículos, por lo que es importante determinar cuál es el modo que consume menos energía por pasajero. Esto se define como intensidad de utilización de energía o consumo específico de energía (figura 1).

Del estudio precitado se extraen una serie de conclusiones:

- El transporte aéreo es el modo de transporte con mayor consumo específico de energía.

- El transporte aéreo consume el doble de energía que el Tren de Alta Velocidad.

- Con una tasa de ocupación del $100 \%$, el ferrocarril y el autobús presentan el consumo específico más bajo.

Este último aspecto es relevante porque durante las horas punta el consumo real de energía de los automóviles es muy alto debido a la congestión del tráfico, además de su escasa

1 Según las estadísticas de Eurostat, el consumo de energía del sector del transporte alcanzó en 1988 el 29,8\% del consumo total de energía final. Esto equivale al consumo de energía de la industria en el mismo ámbito de la Unión Europea.

2 De ese valor, el $55 \%$ corresponde al vehículo privado. 


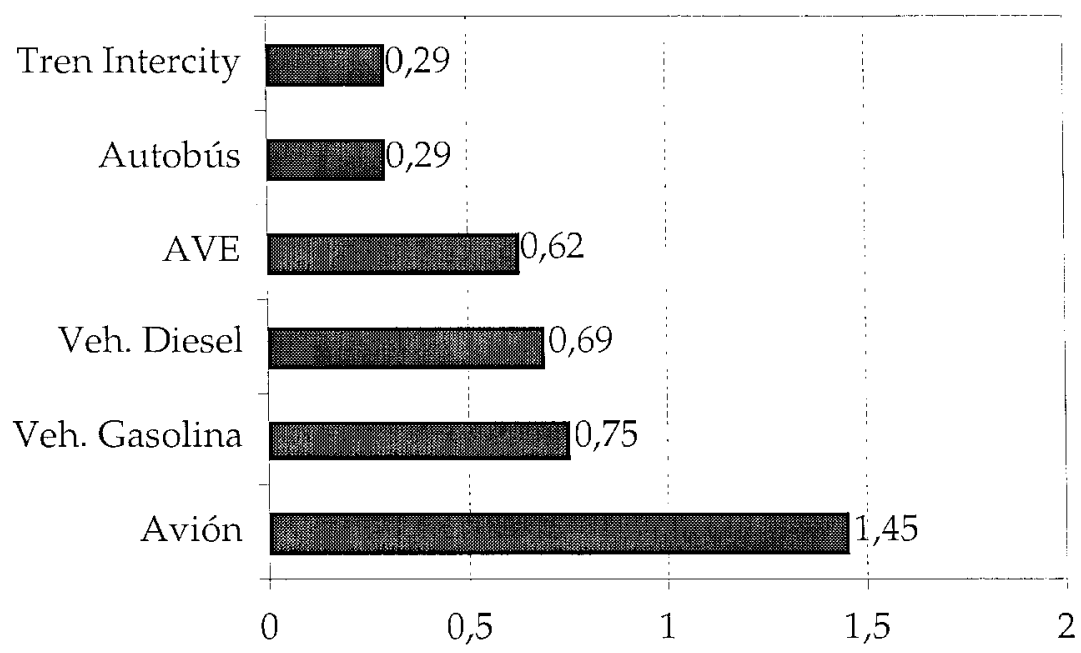

Fuente: MOPT (1992)

Figura 1. Consumo de energía según modos de transporte ( $100 \%$ de ocupación) en megajulios de energía primaria por pasajero-km

ocupación (1 ó 2 personas por vehículo), mientras que el nivel de ocupación del ferrocarril y el autobús es muy elevado, a la par que el consumo de energía es notablemente inferior.

Por otro lado, el consumo de energía no difiere apenas entre el ferrocarril y el autobús; sin embargo, la disponibilidad de plazas, velocidad y fiabilidad hacen más ventajoso el primero. Los valores obtenidos para el transporte de viajeros de los diferentes modos son válidos también para el transporte de mercancías, pues las diferencias entre ellos se mantienen.

En definitiva, el impacto ambiental es la más urgente y principal limitación al desarrollo de los modos de transporte que consumen más energía. Por tanto, la política de transportes debe orientarse en la línea de considerar estas restricciones, favoreciendo el transporte público como modo más eficiente desde el punto de vista energético (ESTEVAN, 1994).

Por otra parte, el transporte es el principal causante de la contaminación total de la atmósfera, de modo que genera la tercera parte del monóxido de carbono, la mitad de los hidrocarburos, óxidos de nitrógeno y plomo, y la cuarta parte de las partículas (LÓPEZ, 1994). Por tanto, esta actividad terciaria supone un factor clave en la alteración de la composición normal del aire.

\section{Tendencias sobre la motorización}

En principio, la importancia del tráfico rodado está in crescendo, de tal modo que las previsiones para el año 2010 en la Unión Europea es de que uno de cada dos ciudadanos 
disponga de vehículo propio. Por ello habrá que motivar al usuario para que opte por un vehículo más respetuoso con el medio ambiente y más eficaz en términos de consumo energético, así como por un uso racional del mismo.

Desde el punto de vista de la motorización, el motor diesel puede continuar per se siendo el dominante en los vehículos pesados y medios ${ }^{3}$, pues no hay una alternativa real a gran escala. Se continuarán haciendo progresos en la mejora de la combustión, la inyección y la puesta a punto de los filtros de partículas. Además, el motor diesel será prioritario en los transportes urbanos, si bien aparecerán aplicaciones puntuales de carburantes de sustitución (BALLESTEROS, 1997).

En cuanto a los turismos y vehículos derivados, el motor de gasolina será el predominante, aunque el poder de penetración del motor diesel crecerá paulatinamente. No hay duda de las ventajas medioambientales del diesel (consumo de combustible, emisiones de $\mathrm{CO}, \mathrm{CO}_{2}$, etc.), pero por sí solo no puede asegurar el $100 \%$ de la motorización por razones de calidad de combustible y equilibrio en las refinerías.

Paralelamente al desarrollo del vehículo eléctrico, debe fomentarse la evolución del vehículo híbrido diesel/eléctrico por razones de ahorro de energía y menor degradación del entorno. En efecto, las prestaciones del motor eléctrico en la ciudad son notables: silencioso, no contamina y velocidad limitada. Por su parte, el diesel aporta importantes ventajas en carretera: escasa emisión de $\mathrm{CO}_{\text {y }} \mathrm{CO}_{2}{ }^{4}$, buena relación consumo/velocidad/aceleración, bajo consumo, fiabilidad y duración. Sin embargo, el principal inconveniente de esta solución es precisamente el coste, por tanto, en esta línea se debe progresar en los años venideros.

Con todo, la investigación del futuro debe estar dictada, a nuestro juicio, por una política de reducción de la contaminación y de ahorro de energía, por lo que las mejoras en la motorización tenderán a conseguir ese objetivo.

\section{IMPACTOS SOCIALES DEL TRANSPORTE}

Aparte de los principales impactos ambientales del transporte, existen otros que tienen un reflejo social de gran trascendencia y que afectan de manera directa a la salud de los seres humanos. Entre ellos se encuentran los accidentes producidos por el tráfico, la ocupación del suelo y la segregación espacial y social.

3 En este sentido, recordemos que en España el 68,8\% de los camiones utilizan el gasóleo como combustible (Ministerio del Interior, 1996)

4 Es importante recordar que Aragón emite a la atmósfera unas $9 \mathrm{Tm}$ por habitante y año de $\mathrm{CO}_{2}$ l lo que equivale a más de la media europea y al doble de la media española. El origen principal de este grado de emisión se localiza en la central térmica de Andorra y en menor medida en las de Escucha y Escatrón. 


\section{Accidentes}

Según estimaciones de la Dirección General de Tráfico, desde mediados de siglo el automóvil se ha cobrado más de 200.000 vidas humanas y ha generado más de tres millones de heridos en España. Por consiguiente, la gravedad social de este fenómeno no admite dudas que supone un punto importante en el discurso de la actividad del transporte.

El hecho de que se produzcan en España en torno 5.000 muertes por año en la década de los $90^{5}$ puede parecer alarmista; sin embargo, es curioso observar cómo las sociedades desarrolladas asumen y aceptan la inseguridad del transporte en su más cruda expresión y sin embargo reaccionan de distinta manera cuando en una catástrofe fallecen unos pocos ciudadanos. Constituye, por tanto, una legitimación social que supera a cualquier otra actividad.

En el caso de Aragón, la siniestralidad supera el 4 \% nacional, lo que supone unos 200 fallecidos por año en nuestras carreteras, de los que más de la mitad están concentrados en la provincia de Zaragoza ${ }^{6}$. Estas cifras de siniestralidad en Aragón son equiparables a su situación general en el marco español, pues sólo tienen valores inferiores las Comunidades Uniprovinciales.

A veces, una manera subliminal de desvirtuar la gravedad de un hecho real es modificar el concepto de los términos. Así nos podemos encontrar con un elemento poco conocido como el de peligro y riesgo, de forma que la Seguridad Vial los utiliza con cierta profusión, pero que en ocasiones se prestan a una incorrecta interpretación.

Es interesante observar esta contradicción en los peatones y ciclistas, habida cuenta que objetivamente no representan peligro para nadie, pero en cambio son extremadamente vulnerables, y debido a que los usuarios son los que registran mayor riesgo de lesiones se dice que son modos de transporte "peligrosos". Todo ello, claro está, frente a los modos de transporte denominados «seguros» (automóvil, ferrocarril, etc.).

Así pues, los accidentes de tráfico son consecuencia del propio desarrollo de la humanidad, pero un desarrollo que comporta gran número de fallecidos que es urgente reducir. Para ello las medidas no deben ir solamente en la línea de educar a los peatones y conductores, sino en la adecuación de los medios de transporte a las características de la circulación, como por ejemplo adaptar la velocidad de los vehículos a la permitida por la normativa vigente.

5 En el año 1996 se produjeron 5.830 muertos, contabilizados a 30 días, Ministerio de Fomento (1997): Anuario Estadístico 1996, Dirección General de Programación Económica y Presupuestaria, 540, Madrid.

6 Por ejemplo, en el año 1996 hubo 174 muertos en Aragón, de los que 112 corresponden a la provincia de Zaragoza, 46 a la de Huesca y 16 a la provincia de Teruel. Por su parte, del global de fallecidos en Aragón, 34 corresponden al ámbito urbano; es decir, el $20 \%$ de los muertos se producen en las ciudades. Ministerio de Fomento (1997). 


\section{La segregación espacial y social}

En ocasiones se ha comparado la situación del automóvil en la ciudad con la de una «bomba», toda vez que la onda expansiva se extiende progresivamente alejando los edificios y las actividades. Pues bien, algo así es lo que ocurre en el interior de las urbes con el desarrollo del transporte, de modo que la ocupación del suelo para el estacionamiento de los vehículos, la red viaria y los equipamientos necesarios exige que las actividades humanas se expandan por el territorio.

Este alejamiento provoca los desplazamientos motorizados que, a su vez, generan más espacio para su utilización. Es decir, se entra en un círculo vicioso donde una necesidad arrastra a la siguiente. Una segunda cuestión es el hecho de que en la ciudad se han creado espacios monofuncionales, en los que predomina una actividad urbana, tales como zona escolar, polígonos industriales, áreas de ocio, áreas comerciales, barrios dormitorio, etc. Esta dispersión de actividades incrementa las distancias entre los diferentes usos y reduce la posibilidad de acceso a pie o en bicicleta; por tanto, esta disposición está forzando el uso de otros modos de transporte.

El resultado de este proceso es lo que SORIA explicó hace unos años (1980), aduciendo que el desarrollo del transporte motorizado sirve paradójicamente para acercar puntos y para alejar usos. Pero para lo que también sirve es sin duda para reducir la propia esencia de las urbes: la convivencia y la comunicación entre los seres humanos.

Es decir, hay que intentar conseguir una ciudad habitable donde existan multiusos que eviten desplazamientos innecesarios en la medida de lo posible. En este sentido parece normal que cuando el AVE se detenga en Zaragoza los viajeros dispongan de medios para alojarse y descansar, en lugar de tener que desplazarse a otras áreas de la ciudad. Por el contrario, no parece razonable que el tiempo que se ahorra un pasajero con el AVE lo invierta en desplazarse a un hotel, amén de los impactos que genera el propio movimiento de los vehículos.

Se podría justificar que la ciudad crece paralela a su evolución demográfica, pero el caso es que en urbes donde la población apenas sufre variación, o bien en otras donde su tasa de crecimiento es exigua, también se realiza el mismo proceso. Esto puede observarse en Zaragoza, por ejemplo, dado que su expansión por el Sur (Montecanal) o por el Norte (ACTUR) en los últimos años no se justifica sólo por el crecimiento demográfico (CALVO, 1995).

Existen, por tanto, otras connotaciones que expliquen este fenómeno amén del volumen de población. Quizá el deseo de mayor espacio para el desarrollo personal, la amplitud de las nuevas áreas residenciales o bien la adaptación a costumbres foráneas, pero cualquiera que sea la explicación, lo cierto es que las ciudades se transforman y el uso del transporte constituye un eslabón inexcusable. 
Otra consecuencia de la motorización en la ciudad es la segregación espacial que opera en la escala de barrio. El tráfico constituye una barrera infranqueable entre las dos aceras de una calle, si bien no es lo más importante la distancia física que supone la separación de la anchura de la calle, sino la separación psicológica y los comportamientos que acarrea.

Un estudio sobre la evolución del comportamiento social nos ilustra que el tráfico llega más lejos que la propia separación espacial. El estudio se basa en la comparación de movilidad de los niños ingleses en 1971 y 1990 (TOLLEY, 1995). El resultado fue que los niños de 7-8 años realizaban solos el desplazamiento al colegio en un $80 \%$ durante el año 1971; mientras tanto, en el año 1990 otros niños de la misma edad lo realizaban en un $9 \%$.

Por supuesto que la independencia de los niños se ha reducido notablemente, pero además los padres se han convertido en chóferes de sus hijos y en transportistas de los alimentos y otros productos necesarios para la familia. Por tanto, está claro que la dependencia del vehículo ha significado algo más que una transformación espacial, y sobre todo ha significado una modificación de las relaciones interpersonales.

Otro aspecto interesante de relieve social es el retroceso de espacio público asignado a la población en beneficio del automóvil y ferrocarril, esencialmente, pues donde antaño los niños podían jugar o las personas adultas se sentaban al socaire de su casa durante las horas de descanso, ahora existe una carretera, un aparcamiento o una vía ferroviaria que entraña riesgo para los ciudadanos, por lo que se han visto obligados a abandonar esos lugares (SANZ, 1994).

\section{CONSIDERACIONES FINALES}

A modo de conclusión, los impactos medioambientales se configuran como una de las más importantes restricciones al desarrollo de los transportes y, por tanto, a la actividad humana. Es incuestionable la preeminencia del transporte por carretera, tanto de pasajeros como de mercancías, pero también es cierto que desde el punto de vista del entorno y del consumo energético se debe priorizar el transporte colectivo.

Las perspectivas de futuro parece que están orientadas a lograr progresos sustanciales en los motores de combustión tradicionales (gasolina y diesel), y de forma muy especial el diesel. Por otro lado, los combustibles llamados de sustitución (gas de petróleo licuado, gas natural comprimido, etanol, metanol, etc.) presentan ventajas significativas, pero poseen notables limitaciones en cuanto a poder calorífico, coste, autonomía, rendimiento, etc. Ahora bien, estos combustibles pueden encontrar aplicaciones limitadas para algún tipo de transporte: autobuses públicos, taxis, etc.

Una cuestión importante en el transporte es conocer los mecanismos establecidos por las instituciones para evitar, reducir o corregir los impactos ambientales, generados tanto 
por las infraestructuras como por la propia actividad del transporte. En su concepción subyace la idea de evitar en gran medida los efectos dañinos al medio ambiente.

Las líneas de actuación son dos: los estudios de impacto ambiental y el proceso de internalización de los costes externos del transporte. El Real Decreto 1131/1988, de 30 de septiembre de 1988, define la Evaluación de Impacto Ambiental (EIA) como un «estudio realizado para identificar, predecir e interpretar, así como para prevenir las consecuencias o efectos ambientales que determinadas acciones, planes, programas o proyectos pueden causar a la salud y al bienestar humanos y al entorno». Así pues, la EIA es un procedimiento administrativo que tiene por objeto la búsqueda de alternativas de construcción de obras públicas y privadas más benignas ambiental y socialmente y, en todo caso, establecer las medidas correctoras oportunas para disminuir los daños inevitables.

Ahora bien, la EIA converge con las externalidades o las consecuencias ambientales y sociales de la actividad del transporte, las cuales no se traducen en un coste económico del causante, sino que repercuten en la colectividad y en el entorno. Este debate ya ha sido manifestado explícitamente en el Libro Verde aprobado por la Comisión Europea (1995), donde se inicia el texto con esta afirmación: «Se comprueba crecientemente que, con las políticas vigentes, las tendencias del transporte son insostenibles».

La manera de solventarlo es el incremento de las tarifas finales del transporte en función de las consecuencias ambientales y sociales que cada modo genera. No obstante, este punto de vista simplista topa con dificultades de diversa índole. Por un lado, la subjetividad de valoración de los daños ambientales y, por otro, la presión ejercida por los propios modos de transporte en el sentido de que, por ejemplo, la carretera paga sobradamente los costes ambientales que genera.

En definitiva, cualquier tasa ecológica debería pasar por las negociaciones de Bruselas, lugar donde convergen los mismos actores que conforman los mecanismos de la regulación convencional. Lo que sí cabe señalar es orientar la demanda del transporte hacia aquellos modos más benignos social y ambientalmente, aunque siempre con la salvedad de que una reducción drástica de consumos de combustible, por ejemplo, quizá comporte una elevada subida de tasas. En cualquier caso es la mejor solución que se vislumbra a medio plazo.

Por otra parte, una tendencia fundamental del transporte es su tratamiento desde el punto de vista de la movilidad «sostenible», la cual trata de reducir los desplazamientos y de fomentar los modos de transporte más respetuosos con el medio ambiente.

A nuestro juicio, además de esos cambios de escenario, el futuro del transporte debe estar arropado de una inversión de valores colectivos, donde sea una virtud permanente el caminar por la ciudad para acceder al trabajo, al colegio, ir de compras o por el simple gusto de pasear y comunicarse. Este valor en alza es el que se propone y para el que se requiere, por supuesto, una nueva perspectiva cultural. 


\section{BIBLIOGRAFÍA}

BALLESTEROS, M. et al. (1997): «Biocarburantes de origen vegetal: una opción limpia para el transporte», Tecnommbiente, 74, Ciemat y Medio Ambiente, Madrid.

BARKE, M. (1986): Transport and Trade, Oliver and Boyd, 249 p., Edinburgh.

CÁDIZ DELEITO, J.C. (1994): «El transporte y la contaminación. Posibles estrategias y soluciones», Ciudad y Territorio, vol II, 100-101, Ministerio de Obras Públicas, Transportes y Medio Ambiente, Madrid.

CALVO PALACIOS, J.L. (1995): «El Eje de desarrollo del Valle del Ebro y las perspectivas del Eje Norte-Sur de Aragón», III Congreso de Economín Aragonesa, 341-374, Zaragoza.

COMISIÓN DE LAS COMUNIDADES EUROPEAS (1995): Libro Verde. Hacin una limpin y eficiente tarifación en el transporte. Politicas para la internalización de los costes externos del transporte en la Unión Europea, COM 691 final, Bruselas.

COMISIÓN DE LAS COMUNIDADES EUROPEAS (1997): Schecma de Developpement del l'espace communautaire, Noordwijk.

ESTEVAN, A. (1994): «Una aproximación a las cuentas del transporte en España, a la luz de la economía ecológica", Ciudad y Territorio, II, 100-101, Ministerio de Obras Públicas, Transportes y Medio Ambiente, 313-333, Madrid.

ESTEVAN, A. Y SANZ, A. (1996): Hacia la reconversión ecológica del transporte en España, Centro de Documentación y Estudios para la Paz, 384 p., Bilbao.

GROUPE TRANSPORT 200 PLUS (1990): Transport in a fast chancing Europe, 7, Bruselas.

LÓPEZ BONILLO, D. (1994): El medio ambiente, Cátedra, 395 p., Madrid.

MARGALEF, R. (1992): Ecologin, Omega, 951 p., Barcelona.

MEADOWS, D.H. et alt. (1985): Los limites del crecimiento. Informe al Club de Roma, Fondo de Cultura Económica, México.

MINISTERIO DE FOMENTO (1997): Anuario Estadístico 1996, Dirección General de Programación Económica y Presupuestaria, 540 p., Madrid.

MOPT (1992): «El Libro Verde sobre el impacto del transporte en el medio ambiente». Documentos. Comunicación de la Comisión al Consejo y Parlamento, Estudios de Transportes, 37, 49-85, Madrid.

MOPTMA (1994): Plan Director de Infraestructuras 1993-2007, 2" edición, Ministerio de Obras Públicas, Transportes y Medio Ambiente, 427 p., Madrid. 
PIÑEIRO PELETEIRO, R. (1990): Comercio y transporte, Síntesis, 140 p., Madrid.

SANZ ALDUAN, A. (1994): "Calmar el tráfico, domesticar el automóvil. Posibilidades de diseño urbano», Cindad y Territorio, 100-101, Ministerio de Obras Públicas, Transportes y Medio Ambiente, 397-409, Madrid.

SORIA Y PUIG, A. (1980): «¿A qué se llama transporte?», Ciuldad y Territorio, 2, Instituto de Estudios de Administración Local, Madrid.

TOLLEY, R. y TURTON, B. (1995): Transport systems, policy and planning. A geographical approach, Longman, 402 p., London.

WOLKOWITSCH, M. (1992): Géographie des transports, Armand Colin, 191 p., París. 